\title{
Weaknesses of a dynamic ID-based remote user authentication scheme
}

\author{
He Debiao*, Chen Jianhua, Hu Jin
}

School of Mathematics and Statistics, Wuhan University, Wuhan, Hubei 430072, China

\begin{abstract}
The security of a password authentication scheme using smart cards proposed by Khan et al. is analyzed. Four kinds of attacks are presented in different scenarios. The analyses show that the scheme is insecure for practical application.
\end{abstract}

Key words: Authentication; Security; Cryptanalysis; Smart card; Attacks.

\section{Introduction}

User authentication is the essential security mechanism for remote login systems in which a password-based authentication scheme is the most commonly used technique to provide authentication between the legal users and the remote server.

In 1981, Lamport [1] proposed a password-based authentication scheme using password tables to authenticate remote users over insecure network. In Lamport's scheme, password table is used to verify the legitimacy of users. In 2000, Hwang et al. pointed out that once the password table was stolen or modified in this scheme, the whole authentication system will be affected [2]. Therefore, they proposed a remote user authentication scheme using smartcard without maintaining a password table. Afterwards, many schemes have been proposed to enhance the security and practicability [3-11].

A common feature among most of the published schemes is that the user's identity is static in

*Corresponding author.

E-mail: hedebiao@163.com, Tel:+0086015307184927,Fax: +008602787817667 
all the transaction sessions, which may leak some information about that user and can create risk of ID-theft during the message transmission over an insecure channel. To overcome this risk, Das et al. proposed a dynamic ID-based remote user authentication scheme [12]. Compared with other authentication schemes, this scheme has many advantages. In the scheme, the server does not maintain any verifier table, and moreover, the scheme is based on the one-way secure hash function, so its realization is simple and reliable. However, Das et al.'s scheme is completely insecure [13]. It failed to protect the anonymity of a user [14] and is susceptible to the impersonation attack [15], and the guessing attack [16].

To overcome the security pitfalls of Das et al.'s scheme, Liao et al. proposed an improved scheme. Misbahuddin et al. [17] demonstrated that Liao et al.’s scheme cannot withstand impersonation attack, reflection attack and is completely insecure as a user can successfully log on to a remote system with a random password.

In 2009, Wang et al. [18] proposed a dynamic ID-based remote user authentication scheme and claimed that their scheme is more efficient and secure than Das et al.'s scheme. However, Khan et al. [19] identify that Wang et al.'s scheme has the following flaws: no provision of user's anonymity during authentication, inability to offer user free choice in choosing his password, vulnerability to insider attack, no provision for revocation of lost or stolen smart card, and does provide session key agreement. To remedy these security flaws, they proposed an enhanced authentication scheme. They claimed their scheme covers all the identified weaknesses of Wang et al.’s scheme and is more secure and efficient for practical application environment.

However, in this paper, we show Khan et al.’s scheme suffers from three weaknesses: 1) inability to protect the user's anonymity; 2) the session-key problem; 3) inefficiency of the double 
secret keys; and 4) other drawbacks. That is, it fails to fully meet the security requirements that this type of scheme should achieve.

The rest of the paper is organized as follows: Section 2 briefly reviews Khan et al.'s scheme,

Section 3 elaborates on the weakness of their scheme, Section 4 concludes this paper.

\section{Review of Khan et al.'s scheme}

The notations used throughout this paper are described as in the following.

- $U_{i}$ : a user.

- $\quad I D_{i}, P W_{i}: U_{i}$ 's identifier, password respectively.

- $\quad S$ : a remote server.

- $\quad x, y: S$ 's secret keys.

- $T_{i}, T_{S}: U_{i}$ 's current timestamp, and $S$ 's current timestamp, respectively.

- $h(\cdot)$ : a hash function.

- $\oplus$ : bitwise XOR operation.

- $\quad \|$ : concatenation operation

Khan et al.'s scheme involves five phases, the registration phase, the login phase, the authentication phase, the password change phase, and the lost smart card revocation phase. We just give the first four phases here, since the last phase has nothing about our analysis.

Registration phase. In this phase, the user $U_{i}$ initially registers with the server $S$.

1) $U_{i}$ chooses his $I D_{i}$ and $P W_{i}$, generates a random number $r_{i}$, and computes $R P W_{i}=h\left(r_{i} \| P W_{i}\right)$. At last, $U_{i}$ sends $\left\{I D_{i}, R P W_{i}\right\}$ to the server $S$.

2) Upon receiving $\left\{I D_{i}, R P W_{i}\right\}, S$ checks the validity of $I D_{i}$. If $I D_{i}$ is not valid, $S$ 
rejects the registration. Otherwise, $S$ computes $I D U_{i}=I D_{i} \| N_{i}, J_{i}=h\left(x \| I D U_{i}\right)$ and $L_{i}=J_{i} \oplus R P W_{i}$, where $N$ equals 0 if $U_{i}$ is a new user, otherwise $N_{i}$ equals 1 . At last, $S$ delivers the smart card containing $L_{i}$ and $y$ to $U_{i}$.

3) Upon receiving the smart card, $U_{i}$ stores $r_{i}$ in the smart card and completes the registration.

Login phase. In this phase, the user $U_{i}$ sends a login request message to the server $S$ whenever $U_{i}$ wants to access some resources upon $S$.

1) $U_{i}$ inserts his smart card into a smart card reader and then inputs his $I D_{i}$ and $P W_{i}$.

2) $U_{i}$ 's smart card generates a random number $d_{i}$ and computes $R P W_{i}=h\left(r_{i} \| P W_{i}\right)$, $J_{i}=L_{i} \oplus R P W_{i}, \quad c_{1}=h\left(T_{i} \| J_{i}\right), \quad A I D_{i}=I D_{i} \oplus h\left(y\left\|T_{i}\right\| d_{i}\right)$ where $T_{i}$ is the current time stamp.

3) $U_{i}$ 's smart card sends the message $M_{1}=\left\{A I D_{i}, T_{i}, d_{i}, c_{1}\right\}$ to the server $S$.

Verification phase. In this phase, the server $S$ verifies the authenticity of the login message requested by the user $U_{i}$.

1) Upon receiving the message $M_{1}, S$ checks the freshness of $T_{i}$. The freshness of $T_{i}$ is checked by performing $T^{\prime}-T_{i} \leq \Delta T$, where $T^{\prime}$ is the time when $S$ receives the above message and $\Delta T$ is a valid time interval. If $T_{i}$ is not fresh, $S$ aborts the current session.

2) $S$ computes $I D_{i}=A I D_{i} \oplus h\left(y\left\|T_{i}\right\| d_{i}\right)$ and checks the validity of $I D_{i}$. If $I D_{i}$ is not valid, $S$ stops the session.

3) $S$ computes $I D U_{i}=I D_{i} \| N_{i}, \quad J_{i}=h\left(x \| I D U_{i}\right)$ and $c_{1}^{\prime}=h\left(T_{i} \| J_{i}\right) . S$ checks if $c_{1}$ equals $c_{1}^{\prime}$. If $c_{1}$ does not equal $c_{1}^{\prime}, S$ stops the session. Otherwise $U$ is authenticated and $S$ computes $c_{2}=h\left(c_{1} \oplus J_{i} \oplus T_{S}\right)$ and the session key $S_{K}=h\left(c_{2} \oplus J_{i}\right)$. At last, $S$ sends 
the message $M_{2}=\left\{c_{2}, T_{S}\right\}$ to $U$ 's smart card.

4) Upon receiving the message, $U_{i}$ 's smart card checks the freshness of $T_{S}$. The freshness of $T_{S}$ is checked by performing $T^{\prime \prime}-T_{S} \leq \Delta T$, where $T^{\prime \prime}$ is the time when $U_{i}$ 's smart card receives the above message and $\Delta T$ is a valid time interval.

5) $U_{i}$ 's smart card computes $c_{2}^{\prime}=h\left(c_{1} \oplus J \oplus T_{S}\right)$ and checks if $c_{1}$ equals $c_{1}^{\prime}$. If $c_{1}$ does not equal $c_{1}^{\prime}, U_{i}$ 's smart card stops the session. Otherwise, $S$ is authenticated and $U_{i}$ 's smart card computes the session key $S_{K}=h\left(c_{2} \oplus J_{i}\right)$.

Password change phase. In this phase, the user $U_{i}$ changes his/her password any time he/she wants.

1) $U_{i}$ inserts his/her smart card into a smart card reader and then inputs his/her $I D_{i}, P W_{i}$ and the new password $P W_{i}^{\prime}$.

2) $U_{i}$ 's smart card computes $R P W_{i}^{*}=h\left(r_{i} \| P W_{i}\right), J_{i}^{*}=L_{i} \oplus R P W_{i}$ and checks if $J_{i}^{*}$ equals $J_{i}$. If $J_{i}^{*}$ does not equal $J_{i}, U$ 's smart card reject the phase.

3) $U_{i}$ 's smart card computes $L_{i}^{*}=J_{i} \oplus R P W_{i}^{*} \oplus h\left(r \| P W_{i}^{\prime}\right)$ and replaces the value $L_{i}$.

\section{Weaknesses of Khan et al.'s scheme}

\subsection{Inability to anonymity}

Khan et al. claimed that their scheme can protect the anonymity of the user. However, we show that the identity of the target user $U_{i}$ may be guessed by another user who is an insider with his or her own password and smart card. Our attack is based on the observation that $A I D_{i}$ is the XOR-ed value of $I D_{i}$ and a digest of other secret components. The problem is that the secret components are commonly shared among all the users. To be more precise, the attack can 
be implemented as follows:

1) The attacker (say, user $U_{j}$ ) eavesdrops the victim's (say, user $U_{i}$ 's) authentication session from which $A I D_{i}, T_{i}$ and $d_{i}$ can be extracted.

2) The attacker computes his own $A I D_{j}$ with $I D_{j}, P W_{j}, d_{i}$ and $T_{i}$, where $d_{i}$ and $T_{i}$ are the captured session.

3) $A I D_{j}=I D_{j} \oplus h\left(y\left\|T_{i}\right\| d_{i}\right)$ can be prepared because $I D_{j}$ and $P W_{j}$ are the attacker's ID and password, respectively.

4) Now the attacker can get $I D_{i}$ by computing $A I D_{i} \oplus A I D_{j} \oplus I D_{j}$.

In the following, why this attack works is demonstrated. $U_{j}$ can control his smart card completely, and certainly he can also control the value of $T_{j}$ and the random number $d_{j}$, then he lets their value be $T_{i}$ and $d_{i}$ separately, and computes

$$
A I D_{j}=I D_{j} \oplus h\left(y\left\|T_{i}\right\| d_{i}\right)
$$

and

$$
\begin{aligned}
& A I D_{i} \oplus A I D_{j} \oplus I D_{j} \\
& =\left(I D_{i} \oplus h\left(y\left\|T_{i}\right\| d_{i}\right)\right) \oplus\left(I D_{j} \oplus h\left(y\left\|T_{i}\right\| d_{i}\right)\right) \oplus I D_{j} . \\
& =I D_{i}
\end{aligned}
$$

In fact, if the adversary can get any legal user's smart card, then he can carry out the attack. The adversary can obtain the secret information $y$ stored in the stolen smart card by monitoring the power consumption [20] or by analyzing the leaked information [21], then he can compute

$$
\begin{aligned}
& A I D_{i} \oplus h\left(y\left\|T_{i}\right\| d_{i}\right) \\
& =I D_{i} \oplus h\left(y\left\|T_{i}\right\| d_{i}\right) \oplus h\left(y\left\|T_{i}\right\| d_{i}\right) . \\
& =I D_{i}
\end{aligned}
$$




\subsection{The session-key problem}

As noted by Blake-Wilson et al. [22], a number of security properties of key agreement have been proposed and the properties have been used to analyze the security of key agreement. The properties include known-key secrecy, unknown key-share resilience, no key control, key-compromise impersonation resilience and perfect forward secrecy. Forward secrecy requires that, if long-term private keys of one or more entities are compromised, the secrecy of previous session keys established by honest entities can be unaffected.

When analyzing the forward secrecy, Khan et al. claimed even if the server's secret keys $x$ and $y$ happens to be compromised, an adversary cannot impersonate legitimate users by using the revealed keys, and concluded their scheme can preserve the forward secrecy of secret keys $x$ and $y$. They made a mistake in understanding the mean of forward secrecy. In fact, their scheme can't provide the forward secrecy. Once the adversary get the value $x, y$ and some ciphertext translated $c$ between the user and the server, he can compute the session key through the following method.

1) The attacker gets the value $I D_{i}$ using the method described above and let $N_{i}$ be zero.

2) The attacker computes $J_{i}=h\left(x \| I D U_{i}\right)$, where $I D U_{i}$ equals $I D_{i} \| N_{i}$.

3) The attacker computes the session key $S_{K}=h\left(c_{2} \oplus J_{i}\right)$.

4) The attacker gets the plaintext $m$ by decrypting $c$ using the session key $S_{K}$. If $m$ is meaningful, the attacker get the correct session key. Otherwise, he lets $N_{i}$ increment by one and repeats steps 2), 3) and 4) until get the correct session key.

From the above description, we can conclude the attacker can get the correct session by testing the possible value $N_{i}$. Then the search space is $|\mathbf{N}|$, where $\mathbf{N}$ is the set of possible $N_{i}$ and 
$|\cdot|$ represents the cardinality of a set.

In fact, even though the attacker can't get the value $I D_{i}$, he can guess the value $I D_{i}$ to carry out the attack, and the search space is $\mid$ ID $|\times| \mathbf{N} \mid$, where ID is the set of possible ID's,. Note that generally $|\mathbf{N}|$ and $|\mathbf{I D}|$ are not very big, and unlike a space for cryptographic key.

\subsection{Inefficiency of the double secret keys}

We can see that the scheme of Khan et al. requires $S$ to keep two keys secret, i.e., the secret key $x$ and $y$. In common sense, it is possible to achieve the user authentication and key agreement service by using only one secret key. Therefore, two secret keys mean more overheads without the security enhancement for the whole authentication system.

\subsection{Other drawbacks}

In the step 2) of password change phase in Khan et al.’s scheme, the smart card checks if $J_{i}{ }^{*}$ equals $J_{i}$. But, in the registration phase, $J_{i}$ is not stored in the smart card. We think Khan et al.'s may make a mistake when design the registration phase and the password change phase. We demonstrate the drawbacks as follows.

\section{- $J_{i}$ is not stored in the smart card}

If $J_{i}$ is not stored in the smart card, then the step 2) of password change phase in Khan et al.’s scheme must be canceled, then Khan et al.’s scheme is vulnerable to the Denial-of-service (DoS) attack.

In password authentication, DoS attack can cause permanent error on authentication by introducing unexpected data during the procedures of authentication. The most vulnerable procedure is the password changing phase since it usually refreshes the data in storage. If an 
attacker can modify the password, or tamper the message containing password with valid data format, the updated password or its related verification data will then be different from what the user expects. The user can never pass the subsequent authentication thereby. In Khan et al.'s scheme, the password changing phase is performed on the user terminal with smart cards, i.e., the user can change his password without communicating with the server [19]. This enhances the security of password changing as no sensitive message needs to be transmitted over the insecure network. Meanwhile, it relieves the overhead of the/a server. However, due to the drawbacks of design, it is still possible to load a DoS attack on password changing in their scheme.

Suppose an attacker temporarily gets access to the user $U_{i}$ 's smart card, he then inserts the card in a terminal device and performs the following operations. He randomly selects two different passwords $P W^{\prime}$ and $P W^{\prime \prime}$ as the old and the new password, respectively. Then he sends a changing password request to the smart card. Since $J_{i}$ is not stored in the smart card, then the smart card will not check if $J_{i}^{*}$ equals $J_{i}$, it just computes $R P W_{i}^{*}=h\left(r_{i} \| P W^{\prime}\right)$, $L_{i}^{*}=J_{i} \oplus R P W_{i}^{*} \oplus h\left(r \| P W^{\prime \prime}\right)$ and replaces the value $L_{i}$.

From then on, $U_{i}$ can never pass the server's password authentication. This is because in the login phase, $U_{i}$ cannot verify the legal server in the second step. Moreover, he cannot be verified by the server in the last step of authentication phase.

\section{- $J_{i}$ is stored in the smart card}

If $J_{i}$ is not stored in the smart card, then the step 2) of password change phase in Khan et al.'s scheme must be canceled, then Khan et al.'s scheme is vulnerable to the password guessing attack.

In Khan et al.'s scheme $r_{i}, J_{i}$, and $L_{i}$ are stored in the smart card after registration. It's easy 
to say that there is the following relation between $r_{i}, J_{i}$, and $L_{i}$ about $P W_{i}$.

$$
\left.J_{i}=L_{i} \oplus h\left(r_{i} \| P W_{i}\right)\right) .
$$

Then the adversary can carry out the off-line password guessing attack using the relation. The detailed description of the attack is as follows. The adversary can obtain the secret information $r_{i}, J_{i}$, and $L_{i}$ stored in the stolen smart card by monitoring the power consumption [20] or by analyzing the leaked information [21]. Then he can carry out the password guess attack using $r_{i}, J_{i}$, and $L_{i}$.

1). The adversary selects a password $P W^{\prime} s$ from a uniformly distributed dictionary.

2). The adversary computes $\left.J_{i}^{\prime}=L_{i} \oplus h\left(r_{i} \| P W_{i}^{\prime}\right)\right)$.

3). A then verifies the correctness of $P W^{\prime} s$ by checking if $J_{i}$ is equal to $J_{i}^{\prime}$.

4). A repeats steps 1,2 , and 3 of this phase until the correct password is found.

\section{Conclusion}

Smart card-based user authentication technology has been widely deployed in various kinds of applications, such as remote host login, withdrawals from automated cash dispensers, and physical entry to restricted areas.

In [19], Khan et al. proposed a password authentication scheme using smart cards and demonstrated its immunity against various attacks. However, by reviewing of their scheme and analyzing its security, four kinds of weakness, i.e., 1) inability to protect the user's anonymity; 2) the session-key problem; 3) inefficiency of the double secret keys; and 4) other drawbacks are presented in different scenarios. The analyses show that the scheme is insecure for practical application. 


\section{Reference}

[1]. L. Lamport, Password authentication with insecure communication, Communications of the ACM 24 (11) (1981) $770-772$.

[2]. M.S. Hwang, L.H. Li, A new remote user authentication scheme using smart cards, IEEE Transactions on Consumer Electronics 46 (1) (2000) 28 - 30.

[3]. W.H. Yang, S.P. Shieh, password authentication schemes with smart cards, Computers \& Security 18 (8) (1999) $727-733$.

[4]. H.M. Sun, An efficient remote user authentication scheme using smart cards, IEEE Transactions on Consumer Electronics 46 (4) (2000) 958 - 961.

[5]. C.C. Lee, M.S. Hwang, W.P. Yang, A flexible remote user authentication scheme using smart cards, ACM Operating Systems Review 36 (3) (2002) 46 - 52.

[6]. J.J. Shen, C.W. Lin, M.S. Hwang, A modified remote user authentication scheme using smart cards, IEEE Transactions on Consumer Electronics 49 (2) (2003) 414 - 416.

[7]. M. Kumar, New remote user authentication scheme using smart cards, IEEE Transactions on Consumer Electronics 50 (2) (2004) 597 - 600.

[8]. W.C. Ku, S.T. Chang, M.H. Chiang, Further cryptanalysis of fingerprint-based remote user authentication scheme using smartcards, IEE Electronics Letters 41 (5) (2005).

[9]. M.K. Khan, J. Zhang, Improving the security of ' 'a flexible biometrics remote user authentication scheme’ , Computer Standards \& Interfaces 29 (2007) 82 - 85.

[10].S.K. Kim, M.G. Chung, More secure remote user authentication scheme, Computer Communications 32 (2009) 1018 - 1021. 518

[11].J.H. Yang, C.C. Chang, An ID-based remote mutual authentication with key agreement 
scheme for mobile devices on elliptic curve cryptosystem, Computers \& Security 28 (2009) $138-143$.

[12].M.L. Das, A. Saxena, V.P. Gulati, A dynamic ID-based remote user authentication scheme, IEEE Transactions on Consumer Electronics 50 (2) (2004) 629 - 631.508

[13].A.K. Awashti, Comment on a dynamic ID-based remote user authentication scheme, Transactions on Cryptology l (2) (2004) 15 - 16.

[14].H.Y. Chien, C.H. Chen, A remote authentication scheme preserving user anonymity, in: International Conference on AINA’ 05, vol. 2, 2005, p. 2005.

[15].W.C. Ku, S.T. Chang, Impersonation attack on a dynamic ID-based remote user uthentication scheme using smart cards, IEICE Transactions on Communication E88-B (5) (2005) 2165 2167.

[16].I. Liao, C.C. Lee, M.S. Hwang, Security enhancement for a dynamic ID-based remote user authentication scheme, in: Proceedings of the National Conference on Next Generation Web Services Practices, 2005, p. 4.

[17].M. Misbahuddin, C.S. Bindu, Cryptanalysis of Liao - Lee - Hwang’ s dynamic ID scheme, International Journal of Network Security 6 (2008) $211-213$.

[18].Y.Y. Wang, J.Y. Kiu, F.X. Xiao, J. Dan, A more efficient and secure dynamic ID-based remote user authentication scheme, Computer Communications 32 (2009) 583 - 585.

[19].M.K. Khan et al., Cryptanalysis and security enhancement of a 'more efficient \& secure dynamic ID-based remote user authentication scheme’, Computer. Communications. (2010), doi:10.1016/j.comcom.2010.02.011

[20].P. Kocher, J. Jaffe, B. Jun, Differential power analysis, Proc. Advances in Cryptology 
(CRYPTO'99), 1999, pp. 388 - 397.

[21].T.S. Messerges, E.A. Dabbish, R.H. Sloan, Examining smart-card security under the threat of power analysis attacks, IEEE Transactions on Computers 51 (5) (2002) 541 - 552.

[22]. Blake-Wilson S, Johnson D, Menezes A. Key agreement protocols and their security analysis. In Proc. the Sixth IMA International Conference on Cryptography and Coding, Cirencester, UK, LNCS 1355, Springer-Verlag, 1997, pp.30-45. 